\title{
Adaptive Compensation of Friction Forces with Differential Filter
}

\author{
Kouichi Mitsunaga, Takami Matsuo
}

\begin{abstract}
In this paper, we design an adaptive controller to compensate the nonlinear friction model when the output is the position. First, we present an adaptive differential filter to estimate the velocity. Secondly, the dynamic friction force is compensated by a fuzzy adaptive controller with position measurements. Finally, a simulation result for the proposed controller is demonstrated.
\end{abstract}

Keywords: nonlinear friction, adaptive controller, fuzzy basis function expansion, adaptive differential filter.

\section{Introduction}

Friction is one of the greatest obstacles in high precision positioning systems. Since it can cause steady state and tracking errors, its influence on the response of the systems must be considered seriously ([10]). Many friction models have been proposed that differ on the friction effects that are modeled in a lubricated contact. These models are divided into two categories: the kinetic and dynamic friction models. The kinetic friction models take into account the friction effects such as the viscous friction, the Coulomb friction, and the Stribeck effect. Another category of friction model includes dynamic friction model that embody the natural mechanism of friction generation such as the LuGre model ([2]).

The coefficients of the various friction-related effects are usually very difficult to measure. A number of methods for friction estimation and compensation have been proposed for these models: adaptive control, joint torque control, learning control, variable structure control, and so on. Friedland et al.[5] proposed a reduced-order nonlinear observer to estimate the velocity-dependent coefficient of the classical nonlinear friction model with velocity measurements. Huang et al.[9] presented an adaptive radial basis function observer to compensate for the effects of the dynamic friction model full-states measurements. Tomei[15] considered the tracking problem for robot manipulators with unknown parameters and dynamic LuGre model using full-states information. Ge et al.[6] presented adaptive controllers by combining neural network parameterization, dual observer for state estimation/stability and adaptive control techniques based on the dynamic LuGre friction model. Sato et al.[14] proposed an adaptive friction compensation method with an $H_{\infty}$ performance using the neural network approximation that is equivalent to the radial basis function approximation or fuzzy basis function approximation. They used the neural network approximation to parameterize the nonlinear characteristics of the dynamic LuGre model. A sliding-mode type error function is introduced that requires full-states measurements. Canudas de Wit et al.[3] designed an observer-based adaptive friction compensation scheme for systems with generalized position/velocity static characteristics based on full stats measurement. The proposed controller guarantees the global asymptotic stability of the tracking error while preserving boundedness of all the internal signals. The nonlinear friction is approximated either by a linear span of set of continuous known function, or by a neural network of bounded basis function. Putra et al.[12] proposed an observer-based friction compensation for a class of the kinetic friction models with known system parameters based on the strictly positive real condition. In the case of partial-states measurements, an adaptive estimator that does not require the strictly positive realness of the plants is needed. Ray et al.[13] presented a non-model-based friction estimation method using extended Kalman-Bucy filtering. The filter is used to estimate a friction force with the full-states measurements. For the classical nonlinear friction model without velocity measurements, Xia et al.[17] employed a velocity observer based on state space nonlinear friction model and designed an adaptive controller that achieved a semi-global asymptotically stability. The velocity observer is a model-based estimator that requires plant parameters to estimate the velocity. Park et al.[11] proposed a non-model-based differential filter of a nonlinear function based on 
the adaptive control theory. The proposed differential filter is applied to estimate the time-derivative of a nonlinear function of the intensity for each pixel to detect moving objects within a scene acquired by a stationary camera. In estimating the friction forces, the differential filter is applicable to estimate the velocity with the position measurements.

In this paper, we design a friction compensator with the position measurement. To begin with, an adaptive differential filter that is a non-model based adaptive algorithm to estimate the derivative of a signal, is designed to estimate the velocity signal. Next, the velocity signal in friction compensator proposed by Canudas de Wit et al.[3] is replaced by the estimate of the adaptive differential filter. Finally, a simulation result of the proposed compensator is presented. From the simulation result, it is shown that the proposed compensator is robust against additive noises.

\section{Adaptive differential filter}

The velocity information is the most important data to estimate and compensate the friction forces. Most of the conventional papers assume that the velocity is measurable. If the velocity information is not available, we have to estimate the velocity signal. Park et al.[11] proposed the adaptive estimator of the time-derivative of an output that is called the adaptive differential filter. Their estimator causes the estimation error in the case of fast time-varying signals. In this paper, we present another adaptive differential filter applicable to fast time-varying signals.

Let $y(t)$ be a measurement signal at a time $t$ and $f(y(t))$ be its nonlinear function. We define $\theta_{T}(t)$ as the derivative with respect to time of the nonlinear function $f(y(t))$, i.e.

$$
\theta_{T}(t)=\frac{d f(y(t))}{d t}=\frac{d f(y)}{d y} \frac{d y(t)}{d t} .
$$

If $\frac{d f(x)}{d x}$ has the inverse $\left(\frac{d f(x)}{d x}\right)^{-1}$, we have

$$
\begin{aligned}
& \dot{y}(t)=\theta_{T}(t) \xi(t), \\
& \xi(t)=\left(\frac{d f(y)}{d y}\right)^{-1} .
\end{aligned}
$$

The problem is defined as follows:

Problem 1. Design a differential filter to estimate the derivative, $\theta_{T}(t)$, of the nonlinear function $f(y(t))$ with available signal $y(t)$ and without any knowledge of its dynamics.

If the signal $\xi(t)$ is available, Problem 1 is equivalent to the estimation problem of the time-varying parameter $\theta_{T}$ in Eq.(2) with the available signals $y(t)$ and $\xi(t)$. It is assumed that the time-varying parameter in (2) is satisfied the following inequality:

$$
\left|\theta_{T}(t)-\theta_{T 0}\right| \leq \varepsilon_{T 0}
$$

where $\theta_{0}$ is an unknown constant, and $\varepsilon_{0}$ is a known constant. Eq.(2) can be rewritten as

$$
\dot{y}=\theta_{T 0} \xi(t)+\varepsilon_{T}(t) \xi(t)
$$

where $\varepsilon_{T}(t)=\theta_{T}(t)-\theta_{T 0}$. We give an adaptive observer as

$$
\dot{\hat{y}}_{T}=-k\left(\hat{y}_{T}-y\right)+\hat{\theta}_{T}(t) \xi(t)-\hat{\varepsilon}_{T}(t) \operatorname{sgn}\left(\hat{y}_{T}-y\right)|\xi(t)|
$$

where $k>0$. Defining the observer error as $e_{T}(t)=\hat{y}_{T}(t)-y(t)$, we obtain the error system as

$$
\dot{e}_{T}=-k e_{T}+\tilde{\theta}_{T}(t) \xi(t)-\hat{\varepsilon}_{T}(t) \operatorname{sgn}\left(e_{T}\right)|\xi(t)|-\varepsilon_{T}(t) \xi(t)
$$


where $\tilde{\theta}_{T}(t)=\hat{\theta}_{T}(t)-\theta_{T 0}$.

The parameter update laws are selected as

$$
\begin{aligned}
& \dot{\tilde{\theta}}_{T}(t)=\dot{\hat{\theta}}_{T}(t)=-\gamma e_{T}(t) \xi(t) \\
& \dot{\hat{\varepsilon}}_{T}(t)=\left|e_{T}(t)\right||\xi(t)|
\end{aligned}
$$

where $\gamma>0$.

We can prove the following lemma:

Lemma 1. Consider the error system (6). The parameter update laws guarantee the stability of the origin of the error system as follows:

$$
e_{T}(t) \rightarrow 0 \quad(t \rightarrow \infty) .
$$

Moreover, if $\lim _{t \rightarrow \infty} \dot{e}_{T}(t)=0$, then $\lim _{t \rightarrow \infty} \tilde{\theta}_{T \varepsilon}(t)=0$, where $\tilde{\theta}_{T \varepsilon}(t)=\hat{\theta}_{T}(t)-\theta_{T}(t)$.

Proof:

The whole system can be written by

$$
\begin{aligned}
& \dot{e}_{T}=-k e_{T}+\tilde{\theta}_{T}(t) \xi(t)-\hat{\varepsilon}_{T}(t) \operatorname{sgn}\left(e_{T}\right)|\xi(t)|-\varepsilon_{T}(t) \xi(t) \\
& \dot{\tilde{\theta}}_{T}=-\gamma e_{T} \xi(t) \\
& \dot{\hat{\varepsilon}}_{T}=\left|e_{T}\right||\xi(t)| .
\end{aligned}
$$

Define a Lyapunov-like function as

$$
V_{1}=\frac{1}{2}\left(e_{T}^{2}(t)+\frac{1}{\gamma} \tilde{\theta}_{T}^{2}(t)+\tilde{\varepsilon}_{T}^{2}(t)\right)
$$

where $\tilde{\varepsilon}_{T}(t)=\hat{\varepsilon}_{T}(t)-\varepsilon_{T 0}$. Its time derivative is given by

$$
\begin{aligned}
\dot{V}_{1}= & e_{T} \dot{e}_{T}+\frac{1}{\gamma} \tilde{\theta}_{T} \dot{\tilde{\theta}}_{T}+\tilde{\varepsilon}_{T} \dot{\hat{\varepsilon}}_{T} \\
= & e_{T}\left(-k e_{T}+\tilde{\theta}_{T}(t) \xi(t)-\hat{\varepsilon}_{T}(t) \operatorname{sgn}\left(e_{T}\right)|\xi(t)|\right. \\
& \left.-\varepsilon_{T}(t) \xi(t)\right)+\frac{1}{\gamma} \tilde{\theta}_{T} \dot{\tilde{\theta}}_{T}+\tilde{\varepsilon}_{T} \dot{\hat{\varepsilon}}_{T} \\
= & -k e_{T}^{2}+\tilde{\theta}_{T}\left(e_{T} \xi(t)+\frac{1}{\gamma} \dot{\tilde{\theta}}_{T}\right)-e_{T} \hat{\varepsilon}_{T} \operatorname{sgn}\left(e_{T}\right)|\xi(t)| \\
& -e_{T} \varepsilon_{T} \xi(t)+\hat{\varepsilon}_{T} \dot{\hat{\varepsilon}}_{T}-\varepsilon_{T 0} \dot{\hat{\varepsilon}}_{T} \\
= & -k e_{T}^{2}-\left(e_{T} \varepsilon_{T} \xi(t)+e_{T} \varepsilon_{T 0} \operatorname{sgn}\left(e_{T}\right)|\xi(t)|\right) \\
= & -k e_{T}^{2}-\left(e_{T} \varepsilon_{T} \xi(t)+\varepsilon_{T 0}\left|e_{T} \| \xi(t)\right|\right) \\
\leq & -k e_{T}^{2}+\left|e_{T}\right|\left|\varepsilon_{T}\left\|\xi\left|-\varepsilon_{T 0}\right| e_{T}\right\| \xi(t)\right| \\
\leq & -k e_{T}^{2} \leq 0 .
\end{aligned}
$$

Hence, we have $e_{T} \in L_{2} \cap L_{\infty}, \tilde{\theta}_{T} \in L_{\infty}, \tilde{\varepsilon}_{T} \in L_{\infty}$. Since $e_{T} \in L_{\infty}, \tilde{\theta}_{T} \in L_{\infty}, \tilde{\varepsilon}_{T} \in L_{\infty}$, we have $\dot{e}_{T} \in L_{\infty}$. From Barbalat's lemma, we conclude that $\lim _{t \rightarrow \infty} e_{T}(t)=0$. Moreover, setting $u$ as

$$
u_{T}=\tilde{\theta}_{T}(t) \xi(t)-\hat{\varepsilon}_{T}(t) \operatorname{sgn}\left(e_{T}\right)|\xi(t)|-\varepsilon_{T}(t) \xi(t),
$$

the error dynamics can be expressed in a first-order system:

$$
\dot{e}_{T}(t)=-k e_{T}(t)+u_{T}(t) .
$$


If $\lim _{t \rightarrow \infty} \dot{e}_{T}(t)=0$, then

$$
\lim _{t \rightarrow \infty}\left(\hat{\theta}_{T}(t)-\theta_{T 0}-\varepsilon_{T}(t)\right)=\lim _{t \rightarrow \infty}\left(\hat{\theta}_{T}(t)-\theta_{T}(t)\right)=0 .
$$

We obtain the estimator for the differential of the signal $y(t)$ by selecting the nonlinear function $f(y(t))$ as the linear function $f(y(t))=y(t)$. In this case, the regressor signal $\xi(t)$ is equal to 1 . The adaptive observer and the update laws are given by

$$
\begin{aligned}
\dot{\hat{y}}_{T} & =-k\left(\hat{y}_{T}-y\right)+\hat{\theta}_{T}(t)-\hat{\varepsilon}_{T}(t) \operatorname{sgn}\left(\hat{y}_{T}-y\right) \\
\dot{\hat{\theta}}_{T}(t) & =-\gamma e_{T}(t), \dot{\hat{\varepsilon}}_{T}(t)=\left|e_{T}(t)\right|
\end{aligned}
$$

where $e_{T}(t)=\hat{y}_{T}(t)-y(t)$. Thus, we get the estimate of the differential of the signal $y(t)$ as

$$
\hat{\dot{y}}=\hat{\theta}_{T}=-\int_{0}^{t} \gamma e_{T}(\tau) d \tau
$$

We call this estimator (11) the adaptive differential filter.

\section{Nonlinear friction model}

Canudas de Wit et al. present a new dynamic friction model that captures dynamic friction effects (the Gahl effect, frictional memory, stick-slip motion) as well as steady state friction effects, including the Stribeck effect $([2,8])$. The interface between two surfaces is modeled by contact between sets of bristles. If $z$ represents the average bristle deflection, $\dot{y}$ the velocity between two surfaces, the friction force $F_{f}$ can be expressed as

$$
\begin{aligned}
F_{f} & =\sigma_{0} z+\sigma_{1} \dot{z}+\sigma_{2} \dot{y} \\
\dot{z} & =-\alpha(\dot{y})|\dot{y}| z+\dot{y} \\
\alpha(\dot{y}) & =\frac{\sigma_{0}}{f_{c}+\left(f_{s}-f_{c}\right) \exp \left\{-\left(\dot{y} / \dot{y}_{s}\right)^{2}\right\}}
\end{aligned}
$$

where $\sigma_{0}$ is the stiffness for deflection angle, $\sigma_{1}$ the damping coefficient for slip, $\sigma_{2}$ the viscous friction coefficient, $f_{c}$ the Coulomb friction level, $f_{s}$ the level of the stiction force, and $\dot{y}_{s}$ the Stribeck velocity.

The system under discussion is the following equation:

$$
M \ddot{y}(t)+F_{f}(t)=u(t)
$$

where $M$ is known and $F_{f}$ is unknown.

As in Canudas de Wit et al.[3], the nonlinear function $\alpha(\dot{y})$ is approximated by FBFE as

$$
\begin{aligned}
& \alpha(\dot{y})=k^{T} \zeta(\dot{y})+\varepsilon_{f} \\
& k=\left[\begin{array}{lll}
k_{1}, & \cdots, & k_{M}
\end{array}\right]^{T} \\
& \zeta(\dot{y})=\left[\begin{array}{lll}
\zeta^{1}(\dot{y}), & \cdots, & \zeta^{M}(\dot{y})
\end{array}\right]^{T}
\end{aligned}
$$

where $\varepsilon$ is the approximation error and $\zeta^{j}$ is defined as

$$
\zeta^{j}(\dot{y})=\frac{\mu_{A^{j}}(\dot{y}(t))}{\sum_{j=1}^{M} \mu_{A^{j}}(\dot{y}(t))} .
$$

where the membership functions are selected as the Gaussian functions:

$$
\begin{aligned}
\mu_{A^{j}}(v) & =\exp \left(-\frac{\left(v-\bar{v}_{j}\right)^{2}}{2 \sigma_{j}^{2}}\right) \\
\sigma_{j} & =\sigma, \quad j=1, \cdots, M .
\end{aligned}
$$

The approximation error is assumed to be bounded and sufficiently small. 


\section{Controller design}

Consider the tracking control problem of system (12) under the assumption that (i) the parameters $M, \sigma_{0}, \sigma_{1}, \sigma_{2}$ are known and the nonlinear function $\alpha(\dot{y})$ in unknown. The desired trajectory $y_{d}$ is smooth enough and bounded. The tracking error and its derivatives are defined as

$$
e=y_{d}-y, \dot{e}=\dot{y}_{d}-\dot{y}, \ddot{e}=\ddot{y_{d}}-\ddot{y} .
$$

\subsection{Adaptive controller with full-states measurements}

We review the adaptive controller proposed by Canudas de Wit et al.[3] with full-states measurements. They presented the internal state observer for the average bristle deflection as:

$$
\begin{aligned}
\dot{\hat{z}} & =\dot{y}-\hat{k}^{T} \zeta(\dot{y})|\dot{y}| \hat{z}+k_{0} \tilde{z}+k_{1} e \\
\tilde{z} & =\frac{m}{\sigma_{1}} \dot{e}+\frac{K_{d}-m \rho}{\sigma_{1}} e+e_{f} \\
\dot{e}_{f} & =-\rho e_{f}+c \rho e
\end{aligned}
$$

where $\hat{z}$ is the estimate of the average bristle deflection and $k_{0}>0, k_{1}>0, c=\frac{K_{p}-\rho K_{d}+m \rho^{2}}{\sigma_{0}}$.

Defining the estimation error of the average bristle deflection as

$$
\tilde{z}=z-\hat{z}
$$

we have the following estimation error equation:

$$
\dot{\tilde{z}}=-\alpha(\dot{y})|\dot{y}| \tilde{z}-\tilde{k}^{T} \zeta(\dot{y})|\dot{y}| \hat{z}-k_{0} \tilde{z}-k_{1} e
$$

where $\tilde{k}=k-\hat{k}$.

The controller is given as follows[3]

$$
\begin{aligned}
u & =m \ddot{y}_{d}+H(p) e+\hat{F}_{f} \\
\hat{F}_{f} & =\sigma_{0} \hat{z}+\sigma_{1} \dot{\hat{z}}+\sigma_{2} \dot{y}
\end{aligned}
$$

where $H(p)$ is the following differentiator:

$$
H(p)=K_{d} p+K_{p}, \quad p=\frac{d}{d t} .
$$

Moreover, we obtain the tracking error equation as

$$
m \ddot{e}+K_{d} \dot{e}+K_{p} e=\sigma_{1} \dot{\tilde{z}}+\sigma_{0} \tilde{z} .
$$

We give the same adaptive update law as in [3]:

$$
\dot{\hat{k}}=-\Gamma \frac{1}{k_{1}} \zeta(\dot{y})|\dot{y}| z \tilde{z} .
$$

\subsection{Adaptive controller with position measurements}

In the position measurement case, we use the estimate $\hat{\dot{y}}$ of the adaptive differential filter instead of the velocity signal $\dot{y}$. They presented the internal state observer for the average bristle deflection as:

$$
\begin{aligned}
\dot{\hat{z}} & =\hat{\dot{y}}-\hat{k}^{T} \zeta(\hat{\dot{y}})|\hat{\dot{y}}| \hat{z}+k_{0} \tilde{z}+k_{1} e \\
\tilde{z} & =\frac{m}{\sigma_{1}} \hat{\hat{e}}+\frac{K_{d}-m \rho}{\sigma_{1}} e+e_{f} \\
\dot{e}_{f} & =-\rho e_{f}+c \rho e
\end{aligned}
$$


where $\hat{\dot{y}}$ is the estimate of the velocity by the adaptive differential filter and $\hat{\dot{e}}=\dot{y}_{d}-\hat{\dot{y}}$. Defining the estimation error of the adaptive differential filter $\varepsilon$ as $\varepsilon=\hat{\dot{y}}-\dot{y}$, we have

$$
\hat{\dot{e}}=\dot{e}-\varepsilon \text {. }
$$

We have the following estimation error equation:

$$
\dot{\tilde{z}}=-\alpha(\dot{y})|\dot{y}| \tilde{z}-\tilde{k}^{T} \zeta(\hat{\dot{y}})|\hat{y}| \hat{z}-k_{0} \tilde{z}-k_{1} e-k^{T} \varepsilon_{0} \hat{z}-\varepsilon
$$

where $\tilde{k}=k-\hat{k}$ and $\varepsilon_{0}$ is caused by estimation error of the adaptive differential filter. Moreover, we obtain the tracking error equation as

$$
m \ddot{e}+K_{d} \dot{e}+K_{p} e=\sigma_{1} \dot{\tilde{z}}+\sigma_{0} \tilde{z}+\left(K_{d}-\sigma_{2}\right) \varepsilon .
$$

The controller is given as follows:

$$
\begin{aligned}
u & =M \ddot{y}_{d}+K_{d} \hat{\dot{e}}+K_{p} e+\hat{F}_{f} \\
\hat{F}_{f} & =\sigma_{0} \hat{z}+\sigma_{1} \hat{z}+\sigma_{2} \hat{\dot{y}} .
\end{aligned}
$$

Replacing the velocity signal by its estimate of the adaptive differential filter, the adaptive update law is given by:

$$
\dot{\hat{k}}=-\Gamma \frac{1}{k_{1}} \zeta(\hat{\dot{y}})|\hat{\dot{y}}| \tilde{z} \hat{z}
$$

\subsection{Simulation example}

Next example is the following LuGre model:

$$
M=10, \sigma_{0}=5, \sigma_{1}=2 \sqrt{5}, \sigma_{2}=0.01, f_{c}=1, f_{s}=1.5, v_{s}=0.1 .
$$

The initial conditions are selected as

$$
y(0)=0, \dot{y}(0)=0.2, z(0)=1 .
$$

The desired reference signals $y_{d}, \dot{y}_{d}, \ddot{y}_{d}$ are given by

$$
y_{d}=3 \sin 0.02 \pi t, \dot{y}_{d}=0.06 \pi \cos 0.02 \pi t, \ddot{y}_{d}=-0.0012 \pi \sin 0.02 \pi t .
$$

The parameters of the fuzzy basis function are selected as follows:

$$
\begin{aligned}
& \sigma_{j}=0.5(j=1, \cdots, 5), \\
& \bar{v}_{1}=-1, \bar{v}_{2}=-0.5, \bar{v}_{3}=0.0, \bar{v}_{4}=0.5, \bar{v}_{5}=1 .
\end{aligned}
$$

The parameters of the adaptive differential filter is given by $k=1, \gamma=0.5$. The simulation parameters of the compensator are selected as follows:

$$
\begin{aligned}
& K_{d}=K_{p}=1 \\
& k_{0}=1, k_{1}=0.1 \\
& \Gamma=50\left[\begin{array}{llllll}
1 & 0 & 0 & 0 & 0 \\
0 & 1 & 0 & 0 & 0 \\
0 & 0 & 1 & 0 & 0 \\
0 & 0 & 0 & 1 & 0 \\
0 & 0 & 0 & 0 & 1
\end{array}\right] .
\end{aligned}
$$



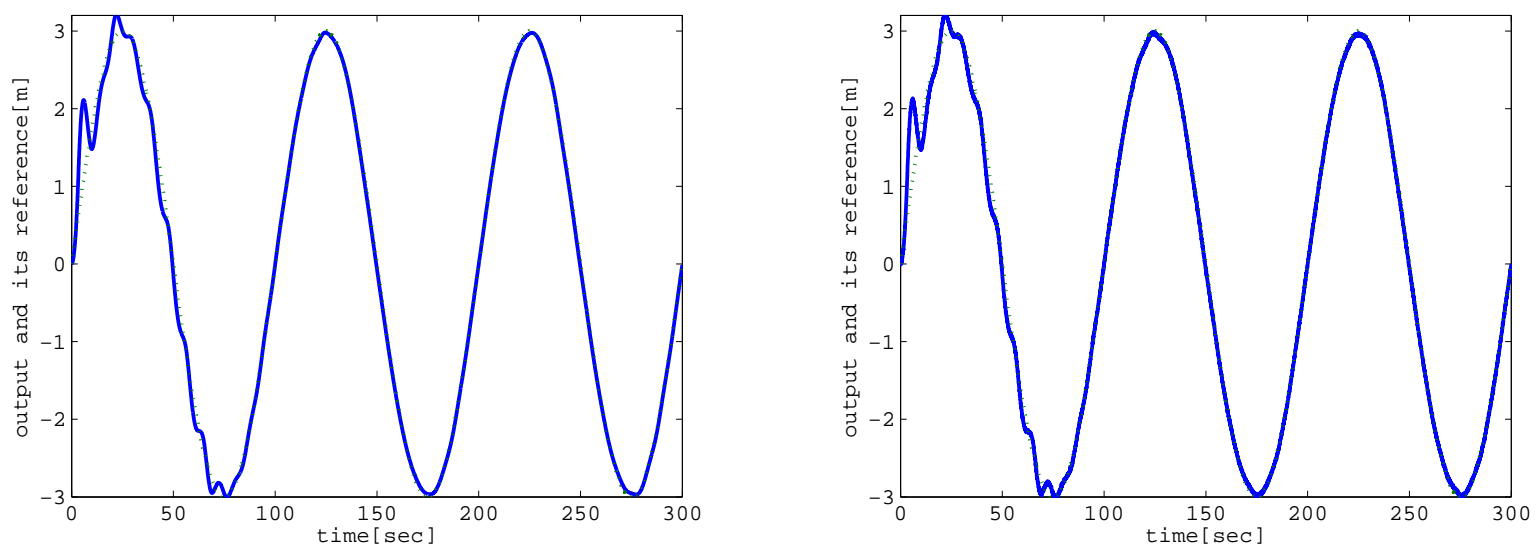

Figure 1: The output $y$ (solid line) and its desired reference $y_{d}$ (dotted line) in the time range $[0,300]$. Left: noise free ,Right: additive noise $N\left(0,10^{-4}\right)$.
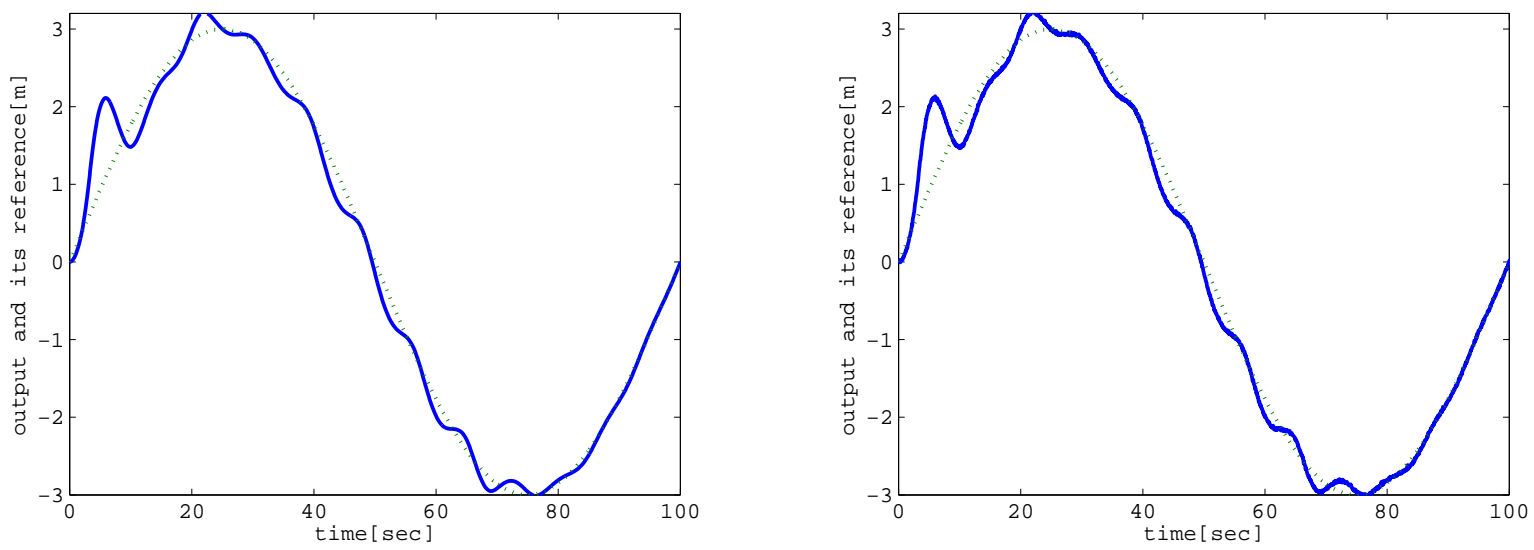

Figure 2: The output $y$ (solid line) and its desired reference $y_{d}$ (dotted line) in the time range $[0,100]$. Left: noise free ,Right: additive noise $N\left(0,10^{-4}\right)$.

The simulation is carried out in the noise free case and in the case where the output $y_{d}$ with the additive noise $N\left(0,10^{-4}\right)$. Figure 1 shows the outputs $y$ (solid line) and its desired references $y_{d}$ (dotted line) in the time range $[0,300]$ in both cases. Figure 2 shows the outputs $y$ (solid line) and its desired references $y_{d}$ (dotted line) in the time range $[0,100]$ in both cases. Figure 3 shows the velocities $\dot{y}$ (solid line) and its estimates $\hat{y}$ by the adaptive differential filter (dotted line) in both cases. Figure 4 shows the inputs $u$ in both cases. Figure 5 shows the friction force(solid line) and its estimate(dotted line). From these figures, the proposed friction compensator is robust against the additive noise.

\section{Conclusion}

We proposed the adaptive differential filter and its application to friction compensation. Specifically, the velocity information in the friction compensator proposed by Canudas de Wit et al.[3] is replaced by the estimate with the adaptive differential filter. From the simulation results, the proposed friction compensator is robust against the additive noise. 

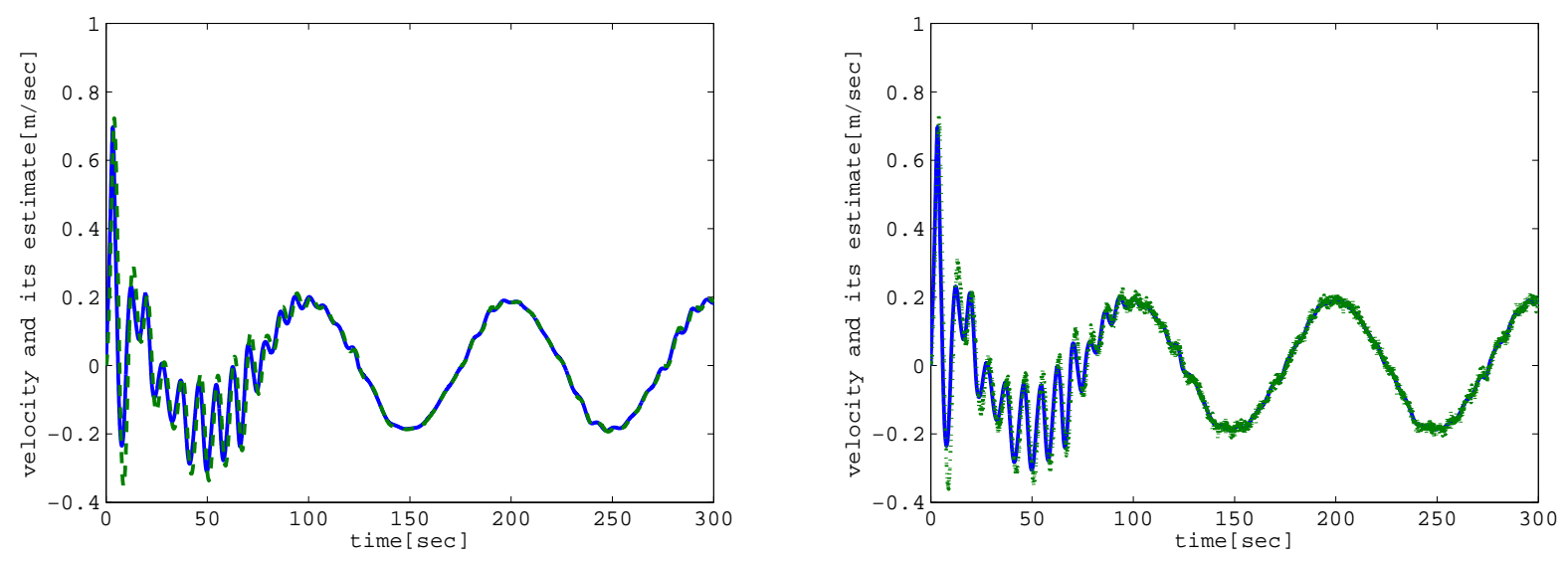

Figure 3: The velocity $\dot{y}$ (solid line) and its estimate $\hat{\dot{y}}$ (dotted line). Left: noise free ,Right: additive noise $N\left(0,10^{-4}\right)$.
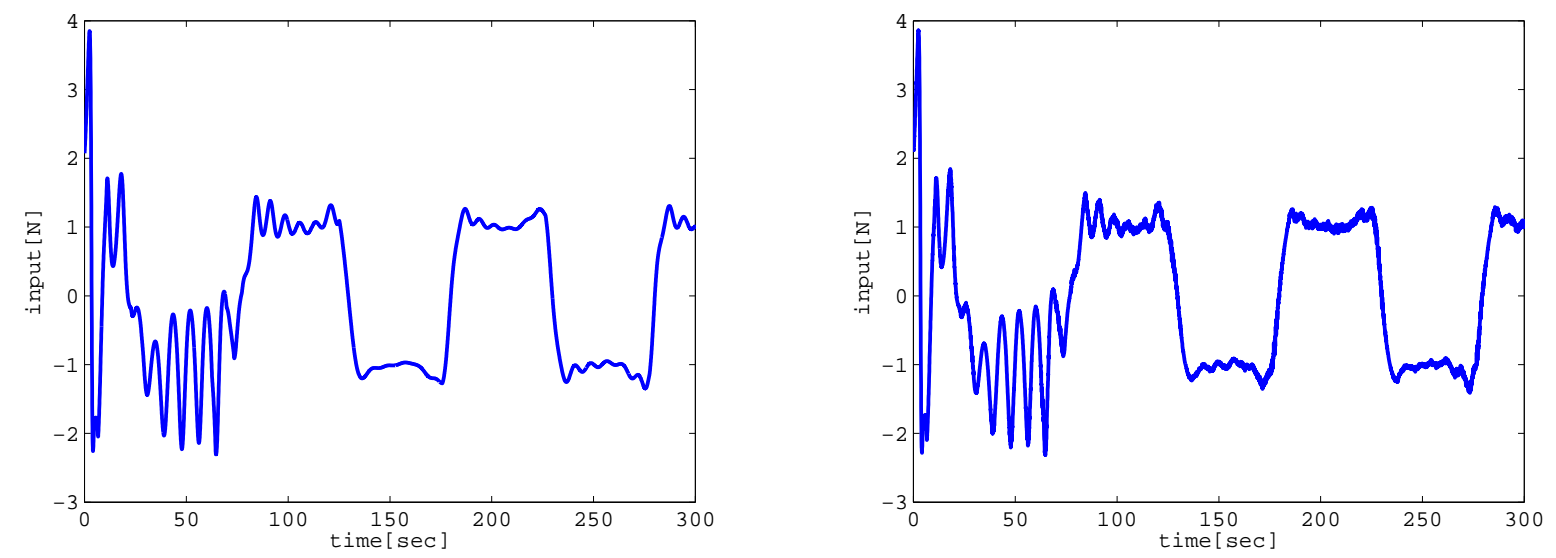

Figure 4: The input signal. Left: noise free ,Right: additive noise $N\left(0,10^{-4}\right)$.
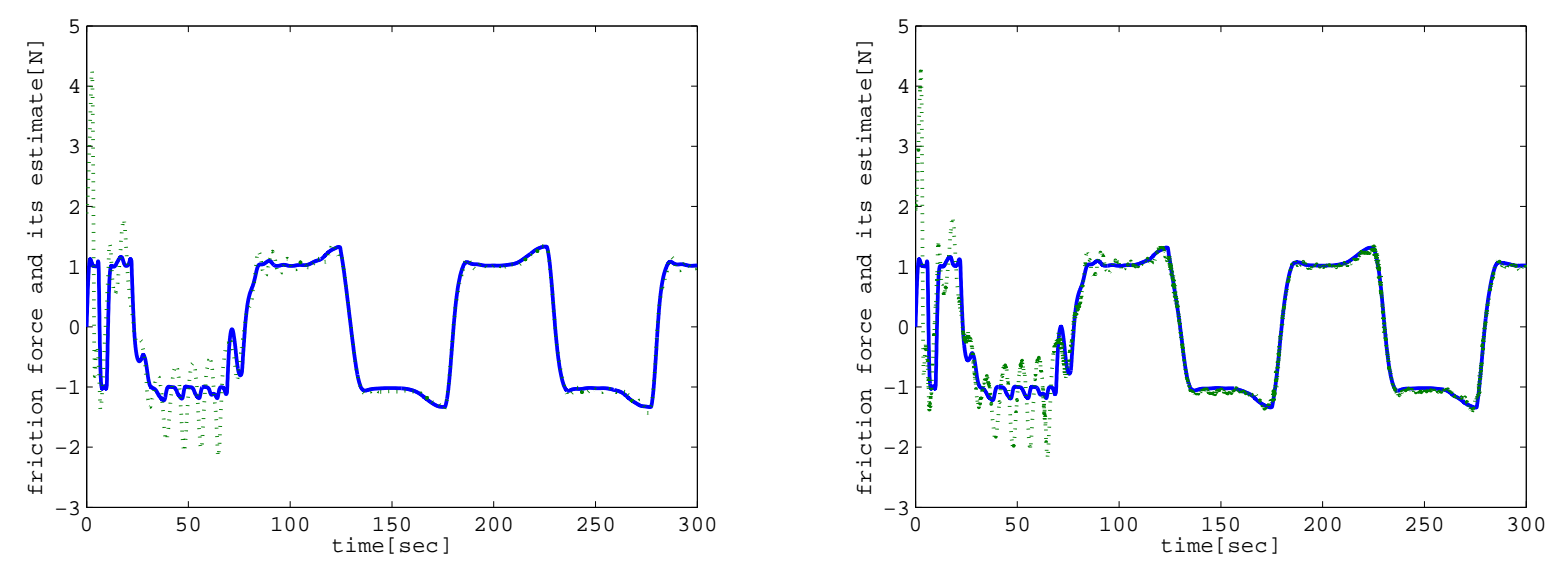

Figure 5: The friction(solid line) and its estimate(dotted line). Left: noise free ,Right: additive noise $N\left(0,10^{-4}\right)$. 


\section{Bibliography}

[1] B. Armstrong-Helouvry, P. Dupont, C. Canudas de Wit, “ A Survey of Models, Analysis Tools and Compensation Methods for the Control of Machines with Friction". Automatica, vol.30, pp.10831138, 1994.

[2] C. Canudas de Wit, H. Olsson, K.J. Astrom, and P. Lischinsky, " A New Model for Control of Systems with Friction”. IEEE Transaction on Automatic Control, vol.40-3, pp.419-425, 1995.

[3] C. Canudas de Wit and S.S. Ge, "Adaptive Friction Compensation for Systems with Generalized Velocity/Position Friction Dependency". Proc. of the 36th IEEE Conference on Decision and Control, pp.2465-2470, 1997.

[4] D. V.-Díaz and Y. Tang, “Adaptive Robust Control of Nonlinear Systems”. IEEE Trans. on Systems, Man, and Cybernetics, Part B, vol.34-3, pp.1596-1601, 2004.

[5] B. Friedland and Y.-J. Park, “ On Adaptive Friction Compensation”. IEEE Transaction on Automatic Control, vol.37-10, pp.1609-1612, 1992.

[6] S.S. Ge, T.H. Lee, and J. Wang, “ Adaptive NN Control of Dynamic Systems with Unknown Dynamic Friction". Proc. of the 39th IEEE IEEE Conference on Decision and Control, pp.1760-1765, 2000 .

[7] H. Han and C.-Y. Su, " Robust fuzzy control of nonlinear systems using shape-adaptive radial basis functions”. Fuzzy Sets and Systems, vol.125, pp.23-38, 2002.

[8] R.M. Hirschorn and G. Miller, "Control of Nonlinear Systems with Friction". IEEE Trans. on Control Systems Technology, vol.7-5, 588-595, 1999.

[9] S.N. Huang, K.K. Tan, T.H. Lee, “ Adaptive motion control using neural network approximations". Automatica, vol.38, pp.227-233, 2002.

[10] E. Papadopoulos and G. Chasparis, "Analysis and Model-based Control of Servomechanisms with Friction”. ASME J. Dynamic Systems, Measurement and Control, vol.126-4, pp.911-915, 2004.

[11] S.-H. Park and T. Matsuo, “ Time-Derivative Estimation of Noisy Movie Data Using Adaptive Control Theory”. International Journal of Signal Processing, vol.2-3, pp.170-177, 2005.

[12] D. Putra, L.P.M. Moreau, and H. Nijmeijer, “ Observer-Based Compensation of Discontinuous Friction". Proc. of the 43rd IEEE Conference on Decision and Control, pp.4940-4945, 2004.

[13] L. R. Ray, A. Ramasubramanian, and J. Townsend, “ Adaptive Friction Compensation Using Extended Kalman-Bucy filter Friction Estimation". Control Engineering Practice, vol.9, pp.169-179, 2001.

[14] K. Sato, Y. Mishima, K. Tsuruta, and K. Murata, “ Adaptive Positioning Control for Linear Slider with Friction Compensation". Trans. of Society of the Instrument and Control Engineers,Japan, vol.40-2, pp.275-277, 2004 (in Japanese).

[15] P. Tomei, “ Robust Adaptive Friction Compensation for Tracking Control of Robot Manipulators". IEEE Transaction on Automatic Control, vol.45-6, pp.2164-2169, 2000.

[16] J. Wang, S.S. Ge and T.H. Lee, "Adaptive Friction Compensation for Servo Mechanism". in: Adaptive Control of Nonsmooth Dynamic Systems G. Tao and F.L. Lewis (Eds.), Springer, pp.211248, 2001. 
[17] Q.H. Xia, S.Y. Lin, M.H. Ang Jr. and T.M. Lim, " Adaptive Joint Friction Compensation Using a Model-Based Operational Space Velocity Observer”. Proc. of IEEE International Conf. on Robotics and Automation, pp.3081-3086, 2004.
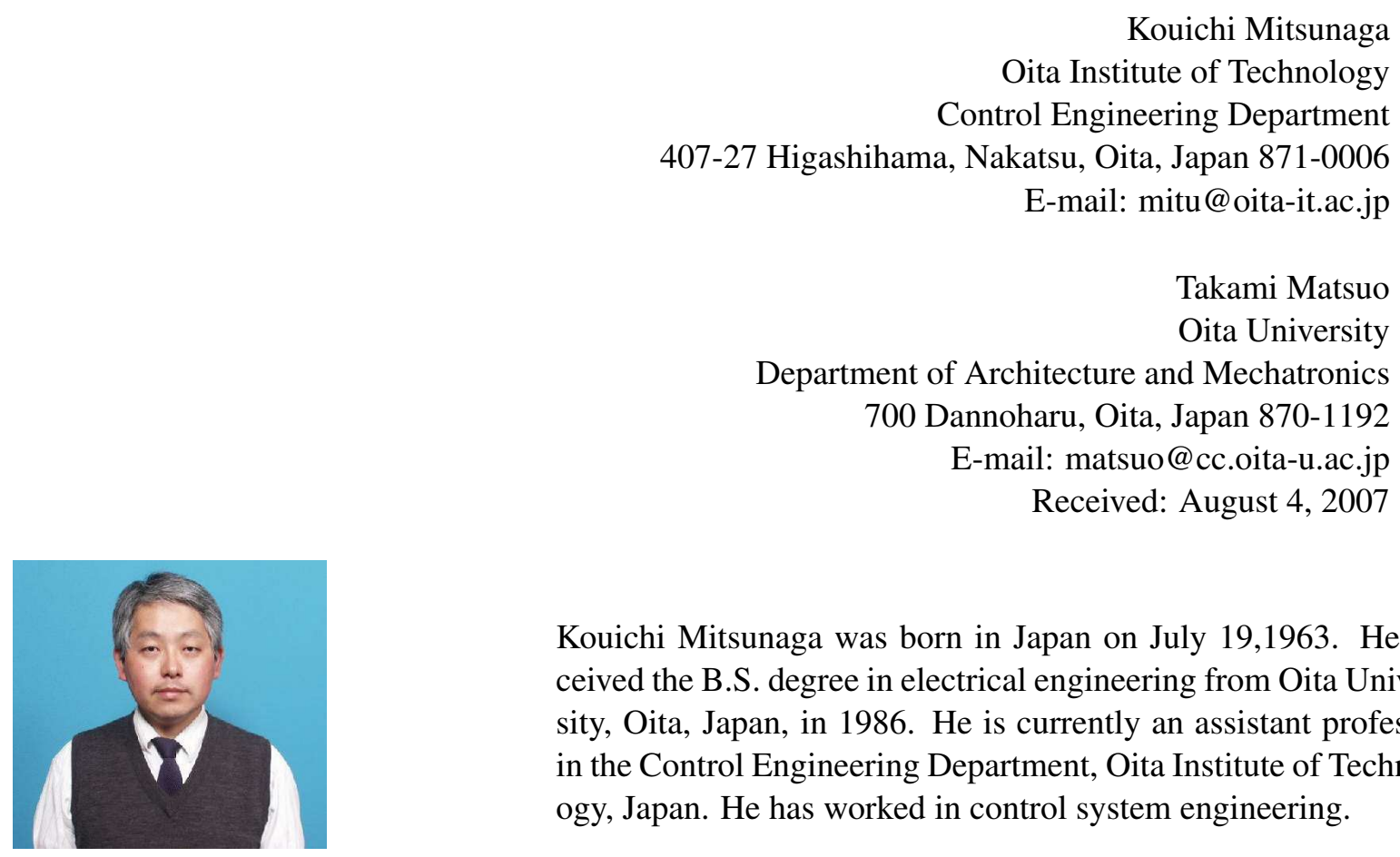

Kouichi Mitsunaga was born in Japan on July 19,1963. He received the B.S. degree in electrical engineering from Oita University, Oita, Japan, in 1986. He is currently an assistant professor in the Control Engineering Department, Oita Institute of Technology, Japan. He has worked in control system engineering.

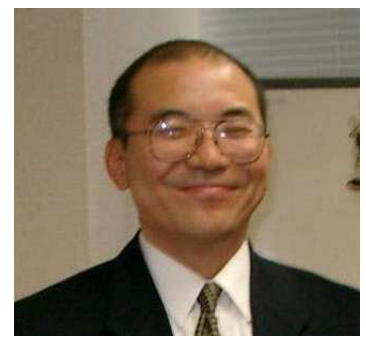

Takami Matsuo was born in Japan on March 24,1958. He received the doctoral degree in engineering from Kyushu University, Fukuoka, Japan, in 1985. He is currently a Professor in the Department of Architecture and Mechatronics, Oita University, Japan. He has worked in control system theory and its applications. Matsuo is a member of IEEE, and SIAM. 